\title{
A NEW QOS GUARANTEE SLA BASED-SCHEME FOR HANDOFF CALLS IN WIRELESS NETWORKS
}

\author{
Nguyen Cao Phuong, Tran Hong Quan
}

Institute of Post and Telecommunication, Post and Telecommunications Institutes of Technologies

\author{
Sang-Ho Lee, Jung-Mo Moon
}

IP Mobility Research Team, Mobile Telecommunication Research Division, ETRI

Received 27 December 2005

\begin{abstract}
The most important thing is guarantee QoS over wireless infrastructures. The efficient of service level agreement (SLA) is becoming increasingly important to both service providers and customers. This paper presents some traffic control schemes for improving QoS, traffic model and performance evaluation are described. We are defining a new scheme for improving handoff call performances in wireless networks, a finite queuing scheme for the handoff calls. SLA measurement calculates the packet delay parameter (PD) of handoff calls. The handoff calls will be accepted into queue if their PD will be smaller than the average waiting time of the queue. Important performance measures of the suggested scheme such as the blocking probability of new call and dropping probability of handoff call are described and evaluated.
\end{abstract}

Keywords: QoS, CAC, SLA, PD, blocking probability, dropping probability.

\section{INTRODUCTION}

As the demand for multimedia services over the air has been steadily increasing over the last few years, wireless multimedia networks have been a very active research area [5, 9]. To support various integrated services with certain QoS requirement in these wireless networks, resource provisioning is a major issue. The wide range of services is characterized by difference bit rates and statistical behavior of multimedia traffic resources and quality of service requirements. Since information loss and transmission delay due to termination of the traffic service even for a very shot time could bring about serious problems.

There are some strategies to reduce network congestion and dropping traffic, call admission control (CAC) is such that. A good CAC scheme has to balance the call blocking and call dropping in order to provide the desired QoS requirements [3, 4, 8, 10]. Also, some control schemes mainly focus on dealing with hierarchical model [1, 9]. Mathematical models for the traffic analysis of mobile communications have been developed [2, 6, 7]. However, all that schemes were for simple voice/data services and some of them for multimedia services [7] but they were too complicate. 
Various handoff priority-based CAC schemes have been proposed [1-7, 11], they can classify into 2 broad categories: Guard channel $(G C)$ scheme - some channels are reserved for handoff call, and Queuing Priority $(Q P)$ scheme - calls are accepted whenever there are free channels. When all channels are busy, new call or handoff call are queued. [4, 6, 10]. Various combinations of the above schemes are possible depending on specific applications. Some services are so sensitive with delay (for example real-time services) and some services are so sensitive with variation of delay.

In order to guarantee end-to-end QoS to customers, service providers have to guarantee the availability and performance of the services and this is increasingly done through SLA. There are few common standards for SLA parameters, the concerning definition of SLA parameters such as availability, reliability, latency, and loss. SLA measurement measure the QoS that service providers deliver to their customers [12]. In our scheme, we use guard channel scheme with finite buffer for handoff calls.

In this paper, we present several traffic control schemes for guarantee QoS in the wireless network for multimedia services. Numerical analysis and performance evaluation in term of new call blocking probability and handoff call dropping probability are described. Section II, system models are described. In section III, performance evaluation study is described. Conclusion of this paper is in section IV.

\section{SYSTEM MODELS}

\subsection{Service level agreement}

Some research has been done on defining SLA parameters. The IP performance working group of the IETF has been working on the identification of Internet service metrics. These internet service metrics are RFC 2330, RFC 2678 etc. To remain competitive, service providers must offer guarantees not only in terms of availability, but also in terms of performance. SLA is specified in traffic conditioning specification and general service characteristics. Some of examples of SLA parameters are described in the following table.

\begin{tabular}{|l|l|}
\hline Parameter & VoIP \\
\hline Throughput & Based on 5 minute collection period at the destination \\
\hline Utilization & $\begin{array}{l}\text { Computed by dividing the measured throughput by the } \\
\text { contracted throughput expectation }\end{array}$ \\
\hline Packet loss & Less than $0.1 \%$ for a 5 min sample \\
\hline Packet delay (PD) & Less than 150 ms for 5 min sample \\
\hline
\end{tabular}

We consider about the packet delay (PD). The priority will be assigned by PD. Which call satisfy the PD parameter can be accepted to the network.

\subsection{Cutoff priority scheme}

In this scheme, priority is given to handoff calls by assigning $C_{h}$ channel exclusively for handoff calls among the total $\mathrm{C}$ channels in the cell. The remaining $C-C_{h}$ channels are shared with both new calls and handoff calls. When call attempts arrive, they are assigned to channels until the number of busy channels reaches $C-C_{h}$. When the amount of busy channels exceeds 
$C-C_{h}$, new calls are blocked and only handoff calls are accepted. When all channels are busy, all new calls and handoff calls are blocked or dropped.

Let $\lambda_{n}, \mu_{n}, \lambda_{h}, \mu_{h}$ are arrival rate and exponential service time distribution rate of new calls and handoff calls, respectively. We use the same approximation works in [6]. The new call arrival is Poisson with arrival rate $\rho_{n}=\lambda_{n} / \mu_{n}$ and with service rate equal 1 . The handoff call arrival is Poisson with arrival rate $\rho_{h}=\lambda_{h} / \mu_{h}$ and service rate equal 1 too. Let $P_{j}$ is probability at the steady state that has $\mathrm{j}$ busy channels $(j=1,2, \ldots, C)$. Then we have:

$$
\begin{aligned}
& P_{j}= \begin{cases}\frac{\left(\rho_{n}+\rho_{h}\right)^{j}}{j !} P_{0} & j \leq C-C_{h} \\
\frac{\left(\rho_{n}+\rho_{h}\right)^{C-C_{h}} \rho_{h}^{j-\left(C-C_{h}\right)}}{j !} P_{0} & C-C_{h} \leq j \leq C\end{cases} \\
& P_{0}=\left[\sum_{j=1}^{C-C_{h}} \frac{\left(\rho_{n}+\rho_{h}\right)^{j}}{j !}+\sum_{j=C-C_{h}+1}^{C} \frac{\left(\rho_{n}+\rho_{h}\right)^{C-C_{h}} \rho_{h}^{j-\left(C-C_{h}\right)}}{j !}\right]^{-1}
\end{aligned}
$$

\subsection{Priority with reserved channel and queue traffic handoffs based on SLA scheme}

In order to reduce the dropping probability of handoff calls, a buffer for handoff calls with finite length $\mathrm{K}$ is used. We use $C_{h}$ channels for handoff calls, exclusively. The remaining $\left(C-C_{h}\right)$ channels are shared by both new calls and handoff calls. A new call is blocked if the number of busy channel exceeds $\left(C-C_{h}\right)$. If $\mathrm{C}$ channels have been in use, $\mathrm{K}$ handoff calls are buffered. As present above, depend on SLA parameters, which call has packet delay parameter smaller than average waiting time in queue, it will be accepted and we call that handoff call type number 1 and another one is handoff call type number 2. Let $\lambda_{n}$ are arrival rate for new calls, $\lambda_{1}$ is arrival rate for handoff calls, whose PD is satisfied PD parameter in SLA and $\lambda_{2}$ is arrival rate for handoff calls, whose PD is not satisfied PD parameter in SLA. The total handoff calls rate is $\lambda_{h}=\lambda_{1}+\lambda_{2}$ and the total rate of arrival call is $\lambda_{T}=\lambda_{n}+\lambda_{h}$. For simplifying calculate, let new call and handoff call have the same exponential service time distribution $\mu$. And then the total traffic load per channel of the system is $\rho_{T}=\lambda_{T} / \mu$. We use the same assumptions as [4], a hexagonal cell shape is assumed for the system and very large population of mobiles is assumed. With these assumptions, we can treat the base station as an $\mathrm{M} / \mathrm{M} / \mathrm{C} / \mathrm{K}$ priority queueing which has $\mathrm{C}$ channels and finite buffer of size $\mathrm{K}$. Let $P_{j}$ is probability at the steady state that has $\mathrm{j}$ busy channels $(j=1,2, \ldots, C+K)$. Then we have:

$$
P_{j}=\left\{\begin{array}{cl}
\frac{\left(\lambda_{n}+\lambda_{h}\right)^{j}}{j ! \mu^{j}} P_{0} & 1 \leq j \leq C-C_{h} \\
\frac{\left(\lambda_{n}+\lambda_{h}\right)^{\left(C-C_{h}\right)} \lambda_{h}^{j-\left(C-C_{h}\right)}}{j ! \mu^{j}} P_{0} & C-C_{h}+1 \leq j \leq C \\
\frac{\left(\lambda_{n}+\lambda_{h}\right)^{\left(C-C_{h}\right)} \lambda_{h}^{C_{h}} \lambda_{1}^{j-C}}{C ! \mu^{C}(C \mu)^{j-C}} P_{0} & C+1 \leq j \leq C+K
\end{array}\right.
$$




$$
\begin{aligned}
P_{0}=\left[\sum_{j=0}^{C-C_{h}} \frac{\left(\lambda_{n}+\lambda_{h}\right)^{j}}{j ! \mu^{j}}\right. & +\sum_{j=C-C_{h}+1}^{C} \frac{\left(\lambda_{n}+\lambda_{h}\right)^{\left(C-C_{h}\right)} \lambda_{h}^{j-\left(C-C_{h}\right)}}{j ! \mu^{j}} \\
& \left.+\sum_{j=C+1}^{C+K} \frac{\left(\lambda_{n}+\lambda_{h}\right)^{\left(C-C_{h}\right)} \lambda_{h}^{C_{h}} \lambda_{1}^{j-C}}{C ! \mu^{C}(C \mu)^{j-C}}\right]^{-1}
\end{aligned}
$$

The probability of blocking for new call is the sum of the probability that the state number of busy channels in the base station is larger than or equal to $C-C_{h}$ :

$$
P_{B}=\sum_{j=C-C_{h}}^{C+K} P_{j}
$$

And the probability of handoff call is the sum of the probability that the state number of busy channels in the base station is larger than or equal to $\mathrm{C}$ :

$$
P_{H}=\sum_{j=C}^{C+K} P_{j}
$$

The probability of handoff call type 1 is given:

$$
P_{H_{1}}=P_{C+K}
$$

Using Little's formula for a steady-state queueing system, we can obtain the average waiting time of handoff call type 1 , which is successful served as:

$$
E\left[W_{H_{1}}\right]=\sum_{j=C}^{C+K} \frac{(j-C) P_{j}}{\lambda_{1}\left(1-P_{H_{1}}\right)}
$$

\section{EVALUATION AND PERFORMANCE}

Some numerical examples are provided in this subsection. Throughout the analysis, we assume that $C=16, C_{h}=4, K=4$. With the cutoff priority scheme, we assume the new call rate $\lambda_{n}=1 / 15$ and the handoff call rate $\lambda_{h}=1 / 20$. In our scheme, we assume the new call rate $\lambda_{n}=1 / 15$ and the handoff call rate of type 1 as $\lambda_{1}=1 / 25$ and the handoff call rate of type 2 as $\lambda_{2}=1 / 100$ (because the number of call type 2 is smaller than the number of call type 1 ). For simple simulation, we assume the service time of all kinds of call is equal and in range $\mu=\{0.04, . ., 0.5\}$.

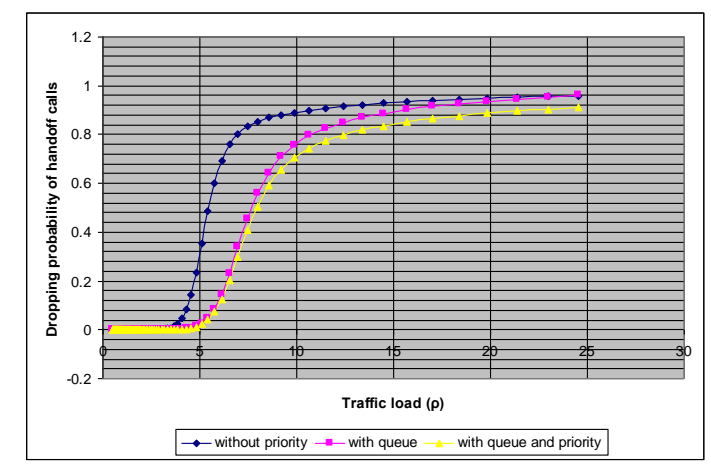

Fig. 1: Dropping probability of handoff calls vs. traffic loads. 


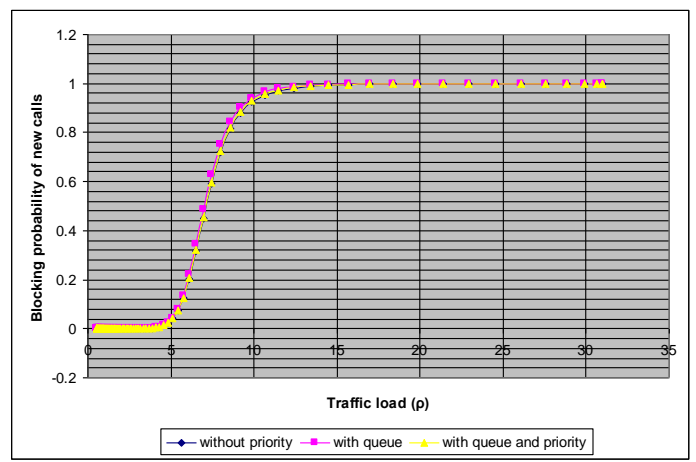

Fig. 2: Blocking probability of the new calls vs. traffic loads.

In Fig. 1, we observe that, as traffic loads increase, the handoff call dropping probability increase too, but our scheme with queue and priority base on SLA has smaller dropping probability. In Fig. 2, the blocking probabilities of new call of all schemes are equal.

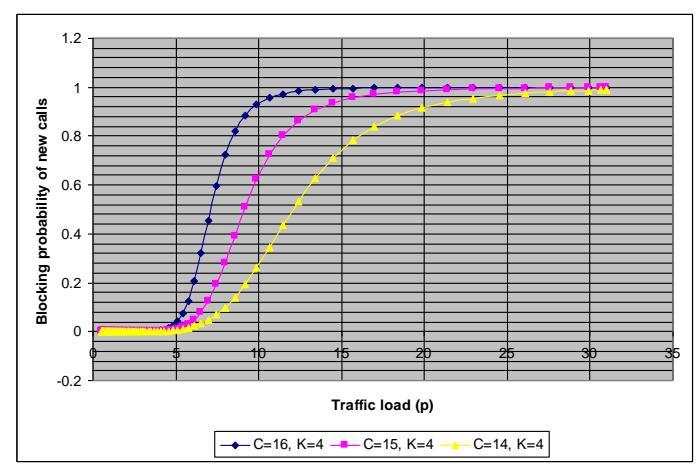

Fig. 3: Blocking probability of the new calls vs. traffic loads with difference $C$

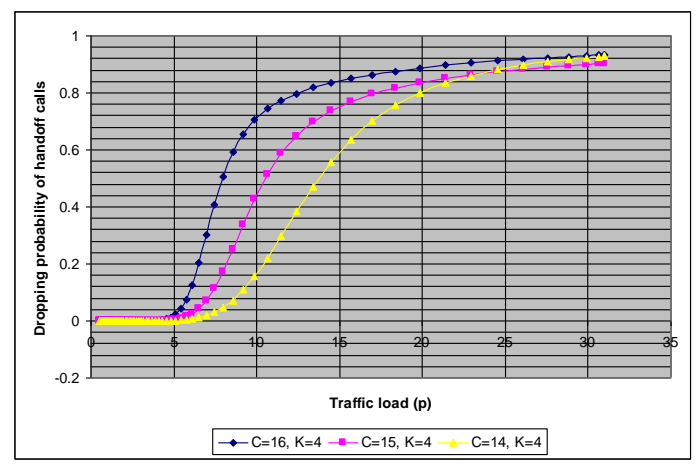

Fig. 4: Dropping probability of the new calls vs. traffic loads with difference $C$.

In Figs. 3 and 4, we observed that the with the same number guard channels and the same buffer and smaller total channels, the dropping probability of new calls and blocking probability of handoff calls are smaller. 


\section{CONCLUSION}

In this paper, we investigate call admission control strategies for wireless networks. We intend to use the SLA parameter to handle multiservice calls, a multiservice call connection may be granted difference QoS levels. In this way, the call traffic will be given resources when SLA parameters are satisfied, hence the desired QoS can be met. This scheme should be useful for the ever increasing multimedia services support with various QoS requirements in the wireless environments.

\section{REFERENCES}

1. Nishith, D. Tripathi, Jeffrey H. Reed, and Hugh F. Vanlandingham (1998), Handoff in Celluar Systems, IEEE Personal Communications, pp. 26-37.

2. Ramachandran Ramjee, Ramesh Nagarajan, and Don Towsley (1997), On optimal Call Admission Control in Cellular Networks, Wireless Network, Vol. 3, pp. 29-41.

3. Guarin, R.A. (1998), Queueing-blocking system with two arrival streams and guard channels," IEEE Trans, Comm., Vol. 36, No. 2, pp. 153-163.

4. Hong, D. and Rappaport, S.S. (1986), Traffic model and performance analysis for cellular mobile radio telephone systems with prioritized and nonprioritized handoff procedure, IEEE Trans. Veh. Technol., Vol.VT-3 5, No. 3, pp. 77-92.

5. Katzela, I. and Naghshineh, M. (1996), Channel assignment schemes for cellular mobile communication systems: A comprehensive survey, IEEE Personal Communications., Vol. 3, pp. 10-31.

6. Fang, Y. and Yi Zhang (2002), Call Admission Control Schemes and Performance Analysis in Wireless Mobile Network, IEEE Transactions on vehicular technology, Vol. 51, No. 2, pp. 371-382.

7. Fang, Y. (2003), Thinning Schemes for Call Admission Control in Wireless Networks, IEEE Transactions on computers, Vol. 52, No. 5.

8. Sang-Ho Lee, Sung-Hee Kim, and Sung-Woo Park (1998), Call Connection Control in CDMA- based Mobile Networks with Multiple Frequency Assignments, Performance of Information and Communication Systems U.Korner \& A. Nilsson (Eds.) IFIP.

9. Ian F. Akyildiz, Janise Mc.Nair, Joseph S.M. Ho, Huseyin Uzunalioglu, and Wenye Wang (1999), Mobility management in Next-generation Wireless Systems, Proceeding of the IEEE, Vol. 87, No. 8, pp. 1347-1384.

10. Sang Gu Choi and Kyoung Rok Cho (2003), Traffic control schemes and performance analysis of multimedia service in cellular systems, IEEE Transactions on vehicular technology, Vol. 52, No. 6.

11. Kailson, J. and Oliver C.Ibe (1995), Cutoff priority scheduling in Mobile Cellular Communication Systems, IEEE Transactions on Communications, Vol. 43, No 2/3/4 February/ March/ April 1995.

12. Jong-Tea Park, Jong-Wook Baek, and James Won-Ki Hong (2001), Management of Service level agreements for Multimedia Internet service Using a Utility Model., IEEE Communications Magazine. 